\title{
Collagen concentrations in dissected tissue compartments of rat uterus on Days 6, 7 and 8 of pregnancy
}

\author{
D. E. Clark ${ }^{1}$, P. R. Hurst ${ }^{1}$, D. B. Myers ${ }^{1}$ and G. F. Spears ${ }^{2}$ \\ ${ }^{1}$ Anatomy Department and ${ }^{2}$ Department of Preventive and Social Medicine, University of Otago, \\ Box 913, Dunedin, New Zealand
}

\begin{abstract}
Summary. Implantation and non-implantation sites were dissected into myometrial and stromal components; a decidual/embryonic region was obtained on Days 7 and 8 of pregnancy. The concentration of collagen (as a percentage of the dry weight of tissue), measured by hydroxyproline analysis, was significantly lower in the implantation regions than in the non-implant regions in all areas studied. The concentrations in the antimesometrial myometrium and stroma of the implantation region remained the same over the days studied. In contrast, the mesometrial collagen concentration in the implantation region declined from Day 6 to Day 8 of pregnancy. Collagen concentration was low within the decidual/embryonic tissue on Days 7 and 8 of pregnancy. Remodelling of collagen within the embryonic area appears to be an important feature of the uterine response to implantation in rats.
\end{abstract}

Keywords: collagen; hydroxyproline; implantation; rat

\section{Introduction}

Embryo implantation in rats is characterized by well-described morphological changes to the uterus (Enders \& Schlafke, 1967; Bell, 1983). A significant uterine change during early implantation is decidualization, which involves the replication and differentiation of certain stromal fibroblasts within the endometrium (Kearns \& Lala, 1983). By Day 6 of pregnancy, the embryo is attached to the luminal epithelium and is found within an implantation chamber. A primary decidual zone can be located close to the embryo (Enders \& Schlafke, 1967; Welsh \& Enders, 1983) and, by Day 7, an essentially avascular primary decidual zone has developed (Welsh \& Enders, 1987; Parr et al., 1988). Welsh \& Enders (1983) noted a bifurcation of the uterine lumen as a non-implantation site becomes incorporated into an implantation site. The development of this antimesometrial lumen assists in the physical isolation of a decidual/embryonic zone on Day 7 of pregnancy. The boundary of the primary and secondary decidual zones is clearly definable on Day 8 (Welsh \& Enders, 1983). At this time, the maternal/embryonic blood system becomes functional in the region that is mesometrial to the embryo (Welsh \& Enders, 1987).

Studies of collagen in the uterus have largely focused on the non-pregnant and post-partum state. Studies of changes in collagen during pregnancy have concentrated on the latter stages (Harkness \& Harkness, 1956). Woessner (1962) found that pregnancy involves a $3 \cdot 5$-fold increase in total uterine collagen between Days 8,9 and parturition, although the concentration of collagen decreases. Wray (1983) measured collagen in the mesentery and in the whole of the uterus during pregnancy and lactation: the results showed a decrease in the concentration of collagen in the uterus and proximal mesentery during implantation. Morphological studies have suggested a decline in collagen in the implantation area (Fainstat, 1963; O'Shea, 1983), but the extent of the decline was not measured. Myers et al. (1990) measured collagen concentration on Days 5, 6, 7 and 11 of pregnancy in rats, comparing whole implantation and non-implantation sites. The results 
showed that from Day 6 collagen concentration was significantly lower in implantation than in non-implantation areas of the uterus.

The present study determined the changes in collagen concentration on Days 6,7 and 8 of pregnancy by assays of separated areas of myometrium, stroma and the decidual/embryonic regions, to gain a better understanding of the temporal and spatial remodelling of the extracellular matrix that occurs in these major uterine tissue compartments during early pregnancy.

\title{
Materials and Methods
}

\begin{abstract}
Animals and treatment. Virgin Wistar rats were supplied by the Animal Breeding Station, Otago University, New Zealand, and at 3-7 months of age were mated on the evening of pro-oestrus. Presence of spermatozoa in the smear on the following morning signified Day 1 of pregnancy. Between 11:00 h and 12:00 h on Days 6,7 and 8 of pregnancy, animals were anaesthetized with Nembutal $(60 \mathrm{mg} / \mathrm{kg}$ intraperitoneal; Abbott Laboratories, New Zealand) and injected intravenously with $0.5 \mathrm{~m} !$ of Pontamine Blue (PB). After $20 \mathrm{~min}$, the animal was perfused with $4 \%$ formaldehyde and $0 \cdot 1 \mathrm{~m}$ sucrose.
\end{abstract}

Dissection. Both horns of the uterus were cut out, the mesentery was trimmed and implantation and nonimplantation areas were separated using a razor blade. Tissue was fixed for a further $30 \mathrm{~min}$. Limits of the nonimplantation and implantation regions were delineated by the PB staining. Non-implantation regions at either extreme of the uterus were discarded. The tissue was washed in buffer for at least 15 min before each of the implant and non-implant regions was dissected under a stereomicroscope at $\times 7$ magnification. Dissection was carried out by making an initial longitudinal incision through the myometrium midway between the mesometrial and antimesometrial aspects. On Days 7 and 8 of pregnancy, a decidual/embryonic area was then separated with tweezers from the implantation areas; this was not possible on Day 6 of pregnancy. The remaining myometrium/stroma was cut so that mesometrial and antimesometrial parts were separated. Stromal tissue was removed with forceps and any excess mesentery trimmed away from the myometrium. All tissue was damp-dried and placed in separate Eppendorf tubes.

The non-implantation and implantation areas dissected on Days $6-8$ of pregnancy included mesometrial myometrium, antimesometrial myometrium, mesometrial stroma and antimesometrial stroma. In addition, implantation areas on Days 7 and 8 included a decidual/embryonic component (Fig. 1). Corresponding tissue areas from both horns were pooled for each animal. Five animals were used on each day of pregnancy. Histological sections were taken of whole implantation sites and dissected tissue pieces on all days of pregnancy to assess the accuracy of the dissection technique. Controls testing for the effects of fixation used undissected pregnant uterus, as dissection could not be carried out on fresh tissue. Pooling tissue from 8-12 implantation sites from each animal and the corresponding number of non-implantation sites gave sufficient tissue for an accurate assay for collagen in each tissue region.

Determination of collagen concentration. Tissue was weighed, freeze-dried and re-weighed to determine the dry weight and the percentage of water in the tissue. Hydrolysis of tissue was carried out in $6 \mathrm{M}-\mathrm{HCl}$ at $105^{\circ} \mathrm{C}$ for $14 \mathrm{~h}$. Hydrolysates were stored at $-20^{\circ} \mathrm{C}$ until analysed for hydroxyproline (duplicates of each sample) by the method of Stegmann \& Stalder (1967). Hydroxyproline concentration was multiplied by a factor of 7.46 (Woessner, 1962) to obtain the collagen concentration, which was calculated as a percentage of the dry weight of tissue.

Statistical analysis. Analysis was carried out using Statworks (Cricket Software Inc, PA, USA) and SPSs (Statistical Package for Social Science, SPSS Inc., Chicago). Analysis of the differences between values over the 3 days of pregnancy studied (Table 1), across non-implant and implant tissue regions, were conducted using the Protected Least-Squares Difference Test, where $t$ test values were derived using the error of the mean square and degrees of freedom from the ANOVA (analysis of variance); $t$ tests were used to compare non-implant with implant results (for each tissue area) on each day.

Multivariate analyses of variance (MANOVA) were conducted to compare each non-implant and implant tissue area (all days of pregnancy included) with other relevant non-implant or implant areas.

Experimental procedures on animals were approved by the Committee on Ethics in the Care and Use of Laboratory Animals at the University of Otago.

\section{Results}

Histological preparations of the various dissection areas showed that the dissection technique produced distinct tissue areas. The most variability was seen within the mesometrial myometrium, which contained the connective tissue of the mesentery. Fixation was necessary for accurate dissection, as provisional attempts with fresh tissue were unsatisfactory. Fixation had minimal effects on hydroxyproline analysis when compared with fresh tissue; a similar result has been found by Myers 
et al. (1977). Overall, collagen values were similar to those obtained by Myers et al. (1990), again confirming that the effects of fixation were minimal.

Significant differences in collagen concentration (Table 1) were observed within corresponding areas between non-implantation and implantation sites. There were no significant changes over Days 6, 7 and 8 within each tissue area in non-implantation sites, but in the implant areas there was a more variable response over the days studied. The mesometrial stroma, mesometrial myometrium and decidual/embryonic areas exhibited different concentrations of decreasing collagen concentration over the days studied. In all cases, collagen concentration was significantly lower $(P<0.01)$ in implantation than in non-implantation areas. The non-implantation mesometrial and antimesometrial myometrium did not differ $(P=0.31)$; all other related tissue areas showed significant differences in collagen concentration. There were no statistically significant differences between the water contents of any of the tissue areas.

For each day of pregnancy, concentration of collagen was lower in the mesometrial myometrium of the implantation area than in the non-implantation area; in the non-implant areas, it did not change, but in the implant areas there was a significant decrease by Day 8 .

In the antimesometrial myometrium, the collagen concentration of the implantation areas was lower than that in the non-implantation areas on all days of pregnancy and did not change significantly in either area over the days examined.

Changes in collagen concentration in the mesometrial stroma in the implantation area were large (comparing Days 6-7 with Days 7-8) and highly significant $(P=0.01)$. In all cases, the collagen concentration of non-implant areas was significantly higher than that of the implantation region. The collagen concentration in the antimesometrial stroma in both areas did not change during the 3 days studied, although there was a lower concentration of collagen in the implantation than in the non-implantation areas.

The most striking result was the very low concentration of collagen on Days 7 and 8 in the decidual/embryonic area (Table 1). The area contained both primary and secondary decidual cells as well as the embryo, which was small in relation to the decidual tissue (Fig. 1), being no greater than $\sim 5 \%$ of tissue, as estimated from histological sections. Collagen concentration was significantly less on Day 8 than on Day 7.

\section{Overall collagen concentration}

The results pooled for non-implant and implant areas (Table 1) are not simple averages, but are adjusted for the differing dry tissue weight each area contributes to the whole. The concentration of collagen in the non-implant region did not change significantly from Day 6 to Day 8, but in the implantation region there was a significant decline (Table 1). On all days studied, there was a lower overall concentration of collagen in the implant region than in the non-implant region.

\section{Discussion}

Dissection of the tissue areas was undertaken to separate the major natural tissue divisions. These divisions were clear when dissecting the myometrium from the stroma and when isolating the embryonic/decidual region. There is a somewhat arbitrary division into a mesometrial and antimesometrial component, but this separation was justified for the following reasons. First, two myometrial samples are necessary because the mesometrial compartment contains both the metrial gland and remnants of the mesentery (Bell, 1979). Removal and analysis of this separately means that a purer myometrium region can be obtained antimesometrially. Secondly, in the case of the stroma, the reason for division reflects distinct functional roles, seen most clearly by Day 8 . Thus, the separation allows for an assessment of the collagen changes within the various tissue regions during the process of implantation. A similar approach, involving examination of the various tissue 


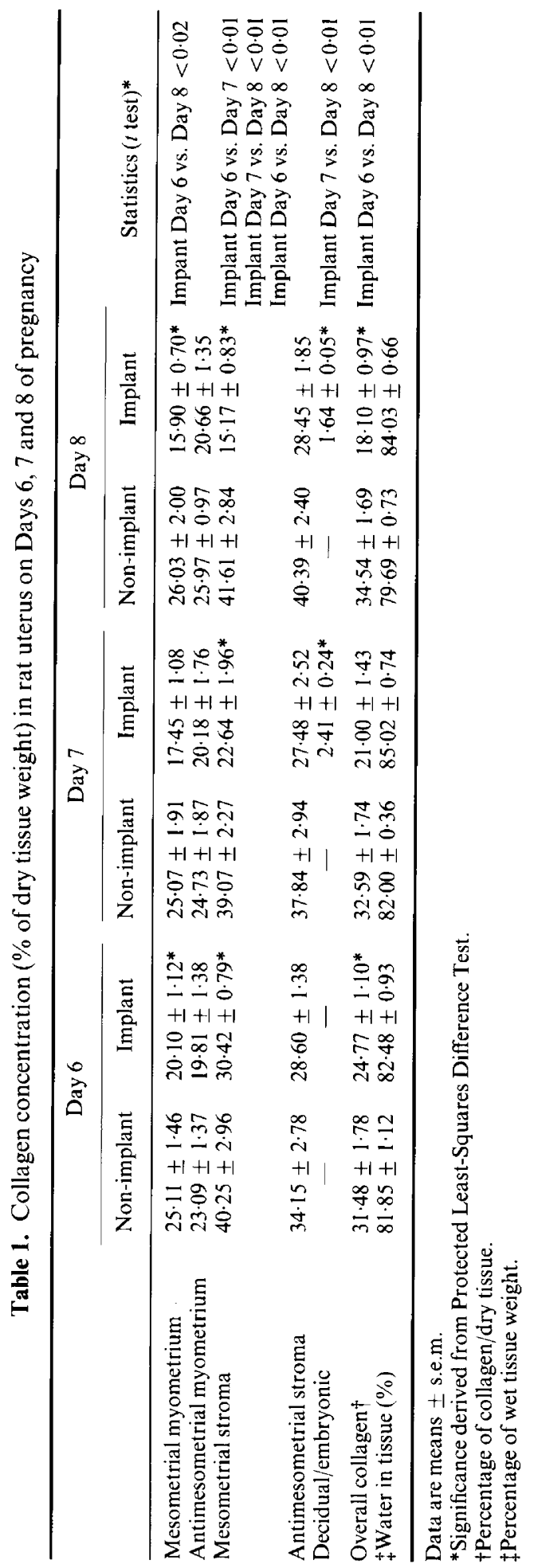




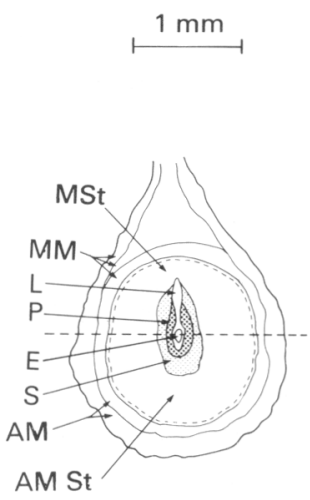

(a) Day 6

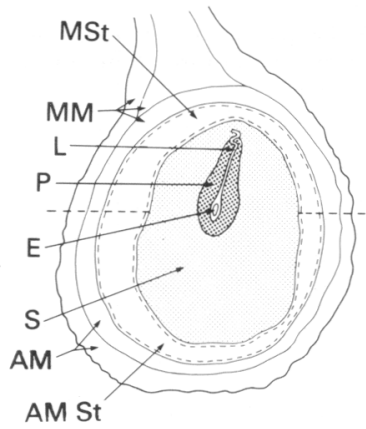

(b) Day 7

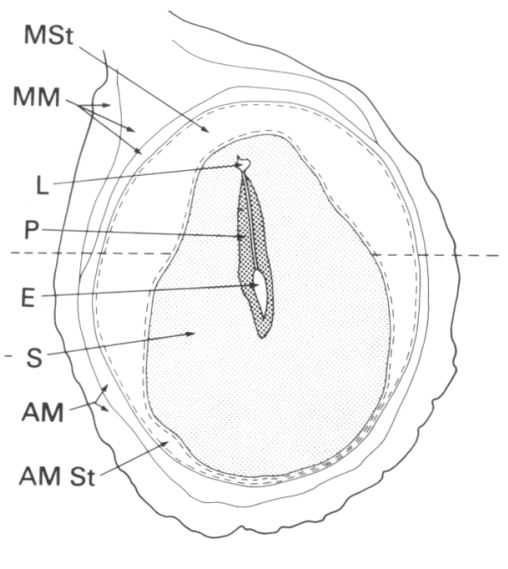

(c) Day 8

Fig. 1. Camera lucida diagrams of transverse sections through rat implantation sites containing the embryo on Days (a) 6, (b) 7 and (c) 8 of pregnancy. Dotted lines indicate the areas of dissection. MSt, mesometrial stroma; MM, mesometrial myometrium; L, lumen; P, primary decidual zone; E, embryo; S, secondary decidual zone; AM, antimesometrial myometrium; AMSt, antimesometrial stroma.

types within rat uterus has been used by De Hertogh et al. (1986) in relationship to hormone receptors and emphasizes the benefits of this approach over studies of whole implantation sites versus non-implantation areas.

Hydroxyproline assays were used to measure the percentage of collagen in the various tissue regions. This assay measures all forms of hydroxyproline in the uterus. Contributions to the hydroxyproline content, other than collagen fibrils, could include collagen peptides, free hydroxyproline, and elastin. Woessner (1962) found that, in the involuting uterus, all other sources of hydroxyproline did not exceed 5\% of the total. Starcher \& Percival (1985) have also shown that, at least in the whole uterus, elastin concentration, and total amount, remains reasonably constant during the early phase of pregnancy. It is thus reasoned that the contribution of elastin, despite having a more concentrated effect due to its probable confinement to the myometrial region, is small and would be of minimal significance in terms of influencing the results of the present study.

Collagen concentration was significantly lower in implantation than in non-implantation areas. This may be because (i) of catabolism of collagen by either extracellular proteinases or intracellular digestion; (ii) reduced collagen concentration may reflect an increase in tissue growth through cell division, infiltration of cells, or an increase in the size of cells already present, resulting in a dilution effect on the collagen concentration or (iii) dilution of the extracellular collagen may have been brought about by a non-collagenous matrix component such as hyaluronic acid. A 5-6-fold increase in the concentration of hyaluronic acid has been shown during early pregnancy, although the total amount present is still small relative to collagen (Carson et al., 1987). An important feature of future work will be to assess which, or what, combination of these effects is important. Different tissue areas may control collagen concentration through different mechanisms.

The mesometrial and antimesometrial myometrium component, in the non-implantation region, on Days 6, 7 and 8 of pregnancy showed no statistical difference in collagen concentration. The collagen concentration in the mesometrial myometrium of the implant region was significantly less than that in the non-implant region. Changes in the implant region over the 3 days were significant. The decrease shown within this region follows the trend found in the mesometrial stroma and suggests the possibility that not all of the stromal tissue was removed from the myometrium. If all of the stromal tissue was removed (as was indicated by the histology preparations), the results may be reflecting a metrial gland change which begins around this time (Bell, 
1979), or an actual effect on the myometrium within this region. When considering the response of the myometrial compartment as a whole, a significant finding was the drop in collagen concentration which had already occurred by Day 6 in the implant area. This suggests an embryonic effect very early in pregnancy and in a tissue region physically separate from the embryo. Another possibility is that certain selected regions along the uterus have undergone changes before the embryonic spacing and represent receptive areas within the uterus. Once again, the nature of the myometrial change is uncertain and may be due to an increase in cell volume or a decrease in total collagen. Further studies of the myometrial tissue in the implantation region are needed.

Mesometrially, the collagen concentration of the stroma in the implantation sites continued to decrease over Days 6-8. This was consistent with the results of Harkness \& Harkness (1956), who found that the collagen concentration in placental sites on Days 8-21 was lower than that in the rest of the uterus. The antimesometrial stroma of the implantation region did not show the gradual decrease found in the mesometrial region. Maintenance of pregnancy requires the development of a placental region and decreased collagen in the mesometrial stroma of the implantation region is likely to reflect the reorganization of this tissue. The decidual regions represent the areas closest to the implanting embryo. Very low collagen concentration is a consistent feature within the decidual region. Transformation and proliferation of stromal cells resulting in the formation of the decidual zone may contribute to lowering the concentration of collagen relative to the total tissue. This dilution effect may be caused by an increase in numbers and/or size of cells and is not associated with a proportional increase in production of collagen in the extracellular matrix (ECM). Degradation of the collagen may also play an important role in both the decline in collagen concentration and in the maintenance of these very low concentrations.

Collagen concentration in the decidual region decreased from Day 7 to Day 8 by $0 \cdot 77 \%$ collagen/dry wt tissue (Table 1); although small in absolute terms, this represents a $32 \%$ difference. The high consistency of the results within each day is reflected in the very low standard errors, suggesting that the collagen concentration in these regions is under strict control. This change in collagen could be explained either by an altered ratio and/or amount of primary and secondary decidual tissue from Day 7 to Day 8 , or by increased collagen degradation. Possible functional reasons for the very low concentrations of collagen in the decidual region could be related to its proposed role as an immunological barrier (Tung et al., 1986), in vascular reorganization, or in cell differentiation.

The results of this study indicate that collagen reorganization within specific areas of the uterus is dramatic during implantation. The approach used has considerable potential for future work in relationship to ECM changes and other uterine responses. Of particular interest was the discovery that collagen concentrations in all regions have already decreased significantly by Day 6 , and, in several cases, reached a plateau within the implantation compartment. This suggests that there would be considerable benefit in looking at uterine changes on Day 5 of pregnancy as well as investigating how implantation induces the very low collagen concentrations in the decidual/ embryonic compartment found in this study.

We are particularly thankful to Professor V. S. Chadwick of the Wellcome Medical Research Institute for the use of the freeze-drier; also to M. Gould for technical assistance and R. McPhee for artwork. D. E. Clark is a Postgraduate Scholar and D. B. Myers is a Senior Research Fellow supported by the Health Research Council of NZ.

\section{References}

Bell, S.C. (1979) Synthesis of 'decidualization-associated protein' in tissues of the rat uterus and placenta during pregnancy. J. Reprod. Fert. 56, 255 262.

Bell, S.C. (1983) Decidualization: regional differentiation and associated function. Oxf. Rev. Reprod. Biol. 5, 220-271.
Carson, D.D., Dutt, A. \& Tang, J.-P. (1987) Glycoconjugate synthesis during early pregnancy: hyaluronate synthesis and function. Devl Biol. 120, 228-235.

De Hertogh, R., Ekka, E., Vanderheyden, I. \& Glorieux, B. (1986) Estrogen and progesterone receptors in the Downloaded from Bioscientifica.com at 04/26/2023 11:07:26AM via free access 
implantation sites and interembryonic segments of rat uterus endometrium and myometrium. Endocrinology $119,680-684$.

Enders, A.C. \& Schlafke, S. (1967) A morphological analysis of the early implantation in the rat. $A m . J$. Anat. 120, 185-266.

Fainstat, T. (1963) Extracellular studies of uterus. I. Disappearance of the discrete collagen bundles in endometrial stroma during various reproductive states in the rat. Am. J. Anat. 112, 337-370.

Harkness, M.L.R. \& Harkness, R.D. (1956) The distribution of the growth of collagen in the uterus of the pregnant rat. J. Physiol., Lond. 132, 492-501.

Kearns, M. \& Lala, P.K. (1983) Life history of decidual cells: A review. Am. J. Reprod. Immunol. 3, 78-82.

Myers, D.B., Pollock, M. \& Calder, C.S. (1977) The analysis of collagen in glutaraldehyde-fixed, osmicated peripheral nerve. Acta Neuropathol. (Berl.) 37, 7.12.

Myers, D.B., Clark, D.E. \& Hurst, P.R. (1990) Decreased collagen concentration in rat uterine implantation sites compared to non-implantation tissue at days 6-11 of pregnancy. Reprod. Fertil. Dev. 2 , $607-612$.

O'Shea, J.D., Kleinfeld, R.G. \& Morrow, H.A. (1983) Ultrastructure of decidualization in the pseudopregnant rat. Am. J. Anat. 166, 271-298.
Parr, M.B., Parr, E.L., Munaretto, K., Clark, M.R. \& Dey, S.K. (1988) Immunohistochemical localization of prostaglandin synthase in the rat uterus and embryo during the peri-implantation period. Biol. Reprod. 38, 333-343.

Starcher, B. \& Percival, S. (1985) Elastin turnover in the rat uterus. Connect. Tissue Res. 13, 207-215.

Stegemann, H. \& Stalder, K. (1967) Determination of hydroxyproline. Clin. chim. Acta 18, $267-273$.

Tung, H.N., Parr, M.B. \& Parr, E.L. (1986) The permeability of the primary decidual zone in the rat uterus: an ultrastructural tracer and freeze-fracture study. Biol. Reprod. 35, 1045-1058.

Welsh, A.O. \& Enders, A.C. (1983) Occlusion and reformation of the rat uterine lumen during pregnancy. Am. $J$. Anat. 167, 463-477.

Welsh, A.O. \& Enders, A.C. (1987) Trophoblast decidual cell interactions and establishment of maternal blood circulation in the parietal yolk sac placenta of the rat. Anat. Rec. 217, 203-219.

Woessner, J.F. (1962) Catabolism of collagen and noncollagen protein in the rat uterus during post-partum involution. Biochem. J. 83, 304-314.

Wray, S. (1983) The effect of pregnancy and lactation on the mesometrium of the rat. J. Physiol., Lond. 340, $525-533$.

Received 7 December 1990 